\title{
Experimental Investigation of the Supersonic Wake of a Reentry Capsule
}

\author{
Ferry F.J. Schrijer* \\ Delft University of Technology, Kluyverweg 1,2629HS, Delft, The Netherlands \\ Louis M.G.F.M. Walpot ${ }^{\dagger}$ \\ Advanced Operations and Engineering Services Group, De Huygensstraat 34, 2201 DK, Noordwijk, The Netherlands
}

\begin{abstract}
The wake behind an Apollo shaped capsule is investigated in the framework of the 'afterbody heating' topic in the RTO WG043 working group. Measurements are performed by means of schlieren, shadowgraphy and stereo particle image velocimetry (SPIV) and are used for CFD validation purposes. The model geometry is a scaled version of the AS-202. It was found from the shadowgraph results that the angle of attack where separation occurs decreases with increasing Mach number and is not influenced by the Reynolds number. Furthermore, it was possible to correlate the shear layer transition location using $R e_{2, x_{t}}$, which is the Reynolds number based on post normal shock conditions where the length scale is the flow path from stagnation point to transition point. SPIV measurements were obtained at Mach 2 and $0^{\circ}$ and $25^{\circ}$ angle of attack. For the $0^{\circ}$ model, the wake was completely separated while for the $25^{\circ}$ model the wake was partially separated and reattaches half way the model. Overall the PIV data return a quantitative three dimensional description of the velocity field around the capsule.
\end{abstract}

\section{Introduction}

Uncertainties in afterbody heating predictions can be related for given re-entry flight conditions to both the thermochemistry between the vehicle surface material and the fluid, as well as to the actual flow field established around the vehicle. In the comparative assessment of the heat transfer prediction performance of different CFD models against free flight test data, the absence of reliable information on the flow field structure can form an important source of uncertainty which can be up to $200 \%$. Moreover, the effects of flow transition to turbulence and of large scale flow unsteadiness require to be ascertained before proceeding with CFD computations, based on the laminar flow regime or the inclusions of turbulence modeling.

In this view a 'afterbody heating' topic was conceived within working group RTO WG043. This paper gives experimental velocimetry and schlieren/shadowgraphy results for the flow around an Apollo-like capsule that was tested in a range between Mach 2 and 4 . In order to build a high quality experimental data base for CFD comparison stereo particle image velocimetry was used and information is given on the location of shear layer transition. In this paper also a correlation is presented that allows to predict the location of shear layer transition.

\section{Experimental apparatus}

\section{II.A. Flow facility and wind tunnel model}

The flow facility used in the experiments is the TST27 transonic/supersonic blowdown wind tunnel, see Figure 1. It has a $27 \times 28 \mathrm{~cm}^{2}$ test section and features two flexible nozzle walls that allow to continuously vary the Mach number between 0.5 and 4.2. The total pressure in the settling chamber can be varied from 2 bar at Mach 0.5 to 20 bar at Mach 4.2, which results in a unit Reynolds number range from $25 \times 10^{6}$ to

\footnotetext{
*Assistant Professor, Faculty of Aerospace Engineering

$\dagger$ Research Scientis
} 


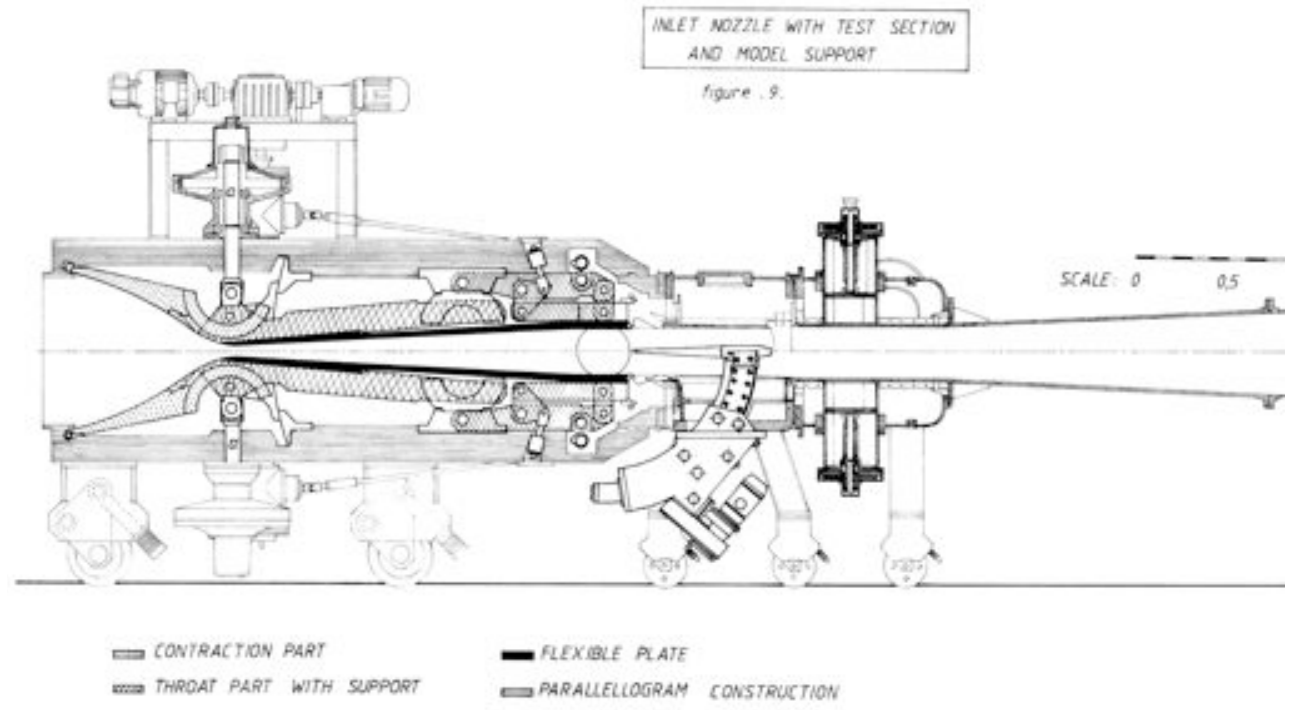

Figure 1. Schematic of the TST27 wind tunnel

$150 \times 10^{6}$. The maximum run-time of the facility is $300 \mathrm{~s}$. Two $30 \mathrm{~cm}$ diameter schlieren windows in the side of the test section are available for optical acces. In the current experiments, the wind tunnel was operated in the range of $M=2-4$ with a total pressure ranging from 2.7-12 bar and a total temperature of $288 \mathrm{~K}$. The capsule geometry used for the definition of the wind tunnel model is a scaled version of the AS-202 outer mold line as defined in Wright et al. ${ }^{1}$ The model has a diameter of $50 \mathrm{~mm}$ and is fabricated out of Makrolon, it is side-mounted on a stainless steel sting. Two models are used for 0 and 25 degrees angle of attack, see Figure 2. For angles of attack that deviated from the 0 or 25 degrees (shadowgraphy measurements), the sting was placed under an angle.

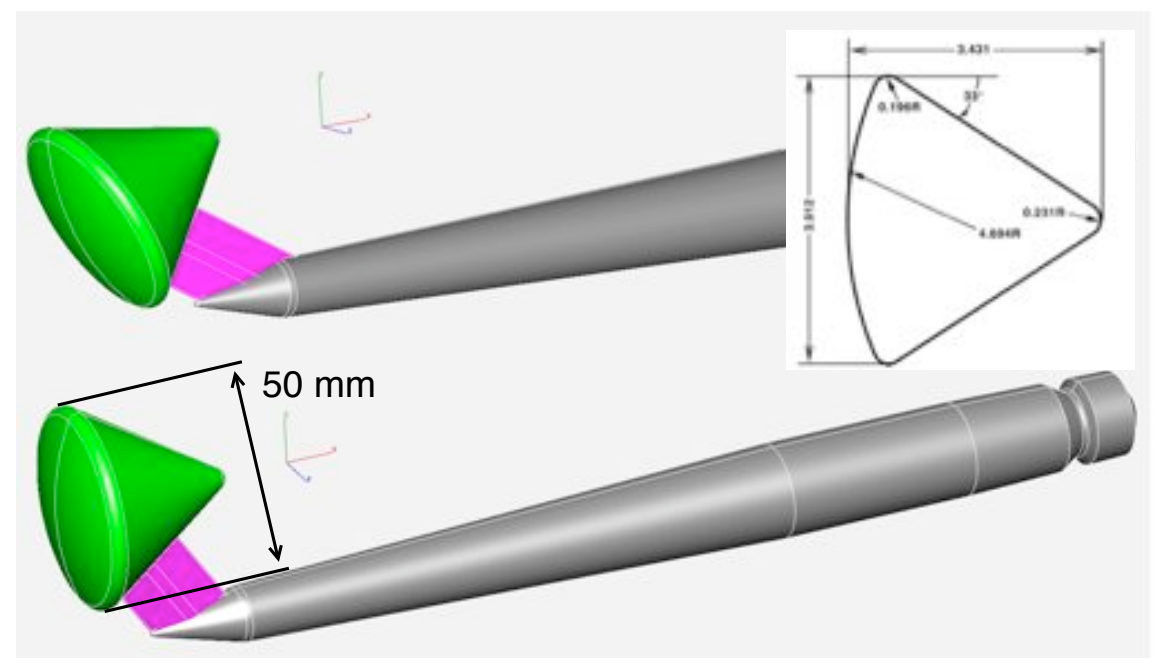

Figure 2. Model and sting geometry for 0 and 25 degree models (insert: model definition by Wright et al ${ }^{1}$ )

\section{II.B. Shadowgraphy and particle image velocimetry}

The shadowgraphs were obtained using a $3872 \times 2592$ pixel Nikon D80 camera. The illumination was provided by a Xenon spark light with an emission time of $20 \mathrm{~ns}$, effectively freezing the flow in a single snapshot. The PIV measurements are performed using a high rep rate illumination and imaging system. A Quantronix Darwin Duo Nd-YLF double pulse laser was used as light source at a repetition rate of $500 \mathrm{~Hz}$. The laser was rated at $20 \mathrm{~mJ}$ per pulse with a duration of $200 \mathrm{~ns}$. The pulse time separation was set to $\Delta t=5 \mu \mathrm{s}$ 
which resulted in a particle displacement of approximately $2.5 \mathrm{~mm}$ between two illuminations. The light was formed into a sheet and introduced into the wind tunnel by means of a retractable probe as shown in figure 3 . The light sheet thickness was approximately $1.5 \mathrm{~mm}$.

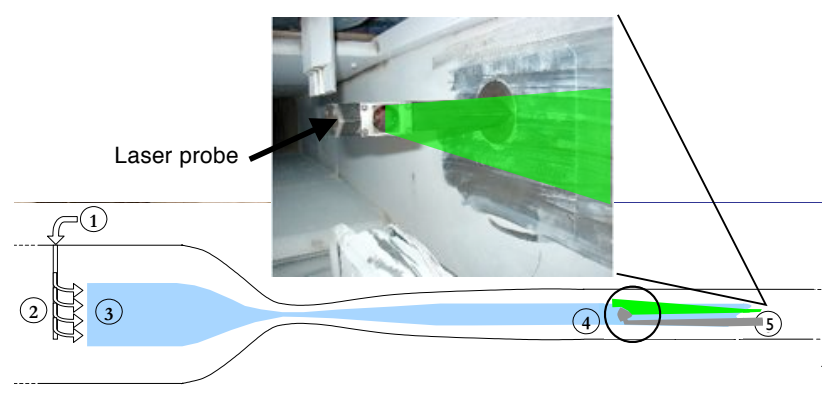

I) Seeding inlet; 2) distributor; 3,4) seeded flow; 5) laser light sheet

Figure 3. Seeding and illumination system

The particle images were recorded by means of two PCO FastCAM cameras which are equipped with a $1024 \times 1024$ pixel CMOS sensor. A Nikkor lens with a focal length of $60 \mathrm{~mm}$ was used at an $f_{\#}=2.8$, furthermore the particle images were slightly defocussed in order to prevent peak locking. For each model two fields of view were considered; the wake region and the 'far' wake region, see Figure 4, each field of view was set to $8 \times 8 \mathrm{~cm}^{2}$. The measurement planes were offset in the $\mathrm{z}$ - direction (symmetry plane) and the following planes were measured: $z=[0,4,7,10,13,16,19,22,25,28,31,34] \mathrm{mm}$. For each plane 500 recordings were used to obtain the velocity data.

PIV measurements were done using a stereo setup which enabled to measure all three velocity components in the plane. ${ }^{2}$ The PIV image interrogation window size was set to $32 \times 32$ pixels with an overlap factor of $75 \%$. This corresponds to a measured vector pitch of $0.7 \mathrm{~mm}$.

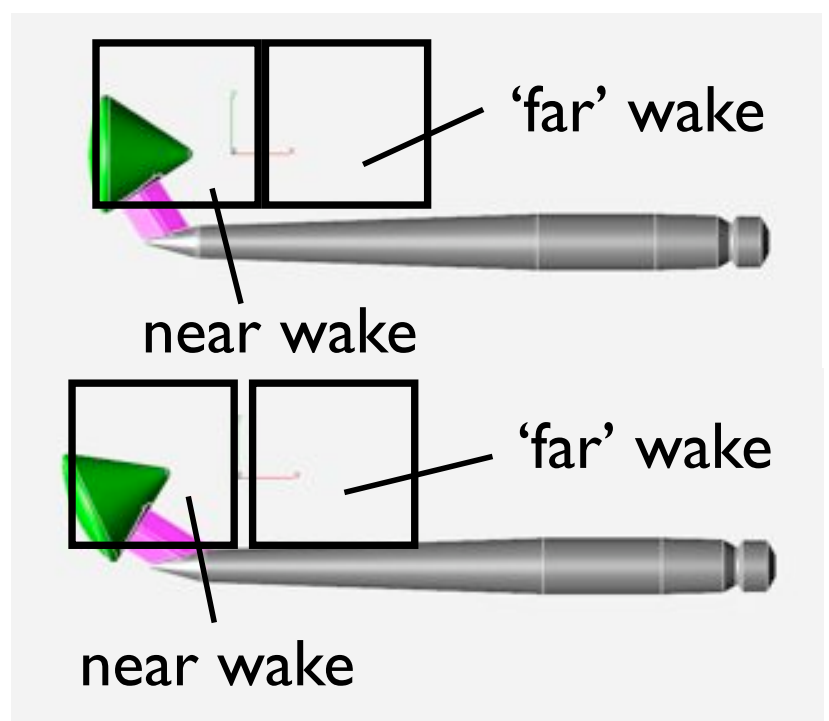

Figure 4. Field of view for the $0^{\circ}$ and $25^{\circ}$ models

\section{Shadowgraphy results}

\section{III.A. Shear layer separation}

Shear layer separation at the capsule shoulder was investigated for various combinations of angle of attack, Mach and Reynolds number. First it was determined wether the flow was attached or separated from the 
wind ward side of the capsule. In Figure 5 an example is given for a separated (left) and attached case (right).
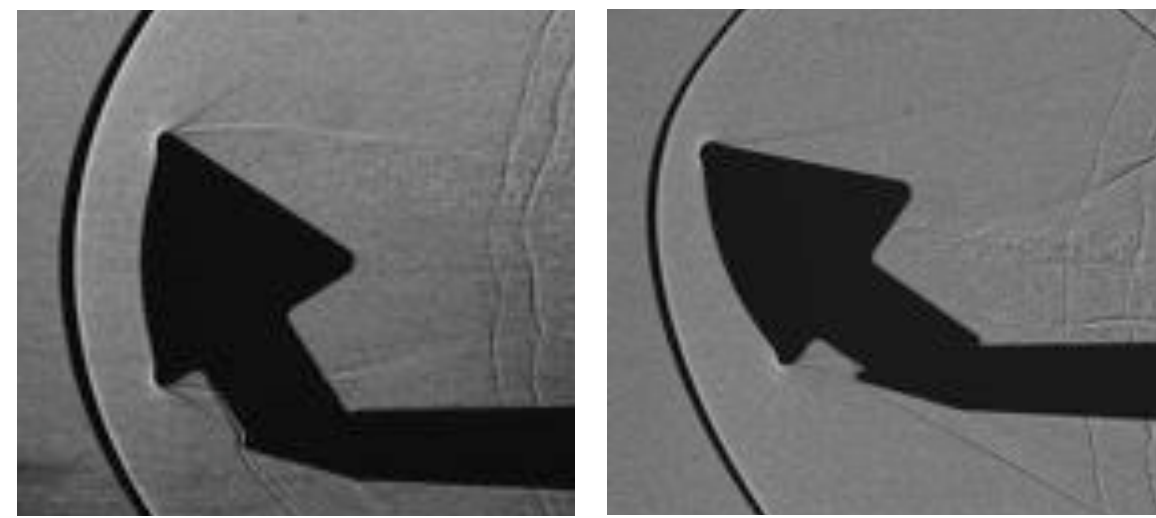

Figure 5. Shadowgraph images for Mach 2, $\operatorname{Re}_{D}=1.75 \times 10^{6}, \alpha=0^{\circ}$ (left) and $\alpha=25^{\circ}$ (right)

It was found that in the current range, the Reynolds number has no effect on separation. Furthermore it was found that for increasing Mach numbers, the flow stays attached for smaller angles of attack. Similar results were obtained by Kruse et al. ${ }^{3}$ In Figure 6, the measurement points are given in angle of attack and Mach number parameter space. The flow conditions for which the shear layer was found to attached are denoted by a blue circle while the separated cases are represented by a red triangle. In the figure also the curve-fit from Kruse et $\mathrm{al}^{3}$ is shown that forms the border between an attached or a separated shear layer. As can be seen, the separation angle of attack decreases with increasing Mach number. However the angle of attack values found by Kruse et $\mathrm{al}^{3}$ are smaller for Mach 2. This is due to the fact that in those measurements models were used that had sharp shoulders, in that case the separation point is defined and a more or less centered Prandtl-Meyer expansion is formed. In the current experiments, the flow expands more gradually and therefore is likely to separate more easily.

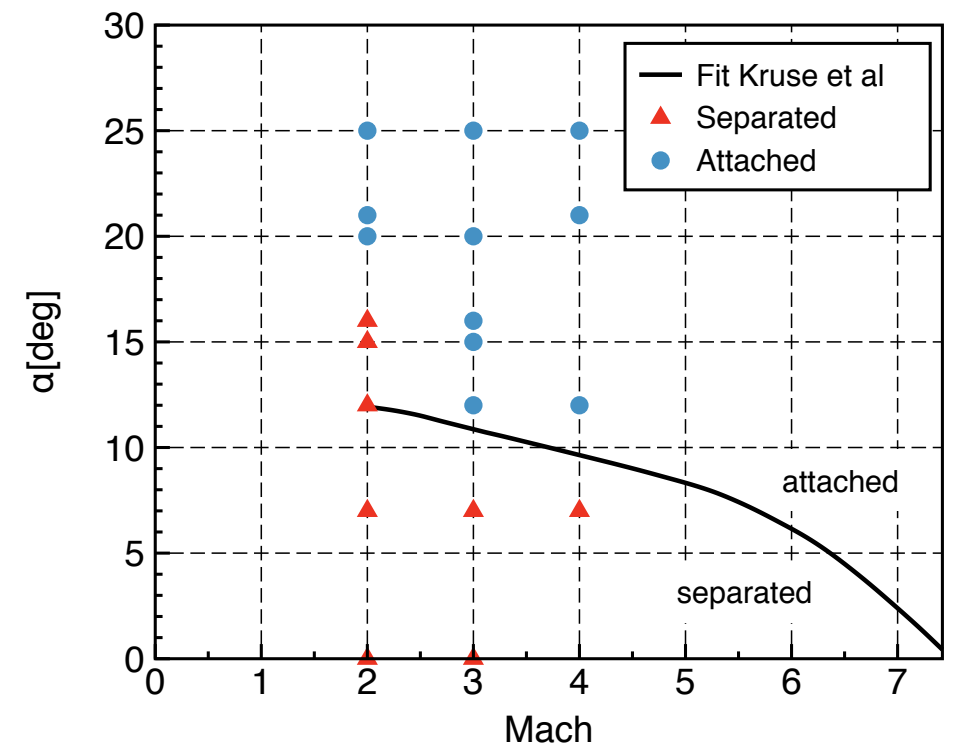

Figure 6. Shear layer condition as a function of Mach number and angle of attack

\section{III.B. Shear layer transition}

The state of the shear layer (laminar or turbulent) is assessed by means of shadowgraph visualization. When the shear layer undergoes the transition from laminar to turbulent, the nature of the flow changes. In Figure 7 a zoomed shadowgraph is shown of the shear layer emanating from the capsule shoulder. The shear layer 
is imaged as a dark-light line. Close near the separation point the line is rather sharp and defined. Moving further downstream, the line becomes less defined and has a more grainy structure. This point has been considered as the shear layer transition location.

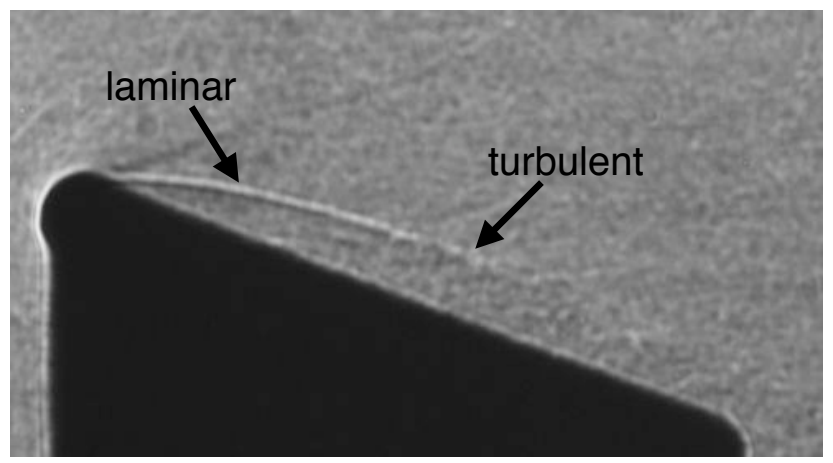

Figure 7. Shadowgraph indicating shear layer transition; Mach 2, $\alpha=15^{\circ}$ and $\operatorname{Re}_{D}=1.75 \times 10^{6}$

The shadowgraphs show that the location of the shear layer transition point depends on $\alpha, M$ and $R e$. It was found that it is possible to take into account within a single parameter: $R e_{2, x_{t}}$. This is the Reynolds number based on the flow conditions downstream of the (normal) bow shock, where the distance from the stagnation point to the shear layer transition location is used as the characteristic length scale:

$$
R e_{2, x_{t}}=\frac{\rho_{2} U_{2} x_{t}}{\mu_{2}}
$$

The Mach number is accounted for by taking the values downstream of the bow shock, therefore taking into account the shock strength. And the angle of attack is taken into account by regarding the length from the stagnation point to the transition point instead of for example the length from the shoulder of the model to the transition point (this was done by Kruse $^{3}$ ). For the current experiments it was found that for all cases : $R e_{2, x_{t}}=0.8 \times 10^{6}$, see Table 1 .

\begin{tabular}{|c|c|c|c|c|}
\hline Mach & $\alpha[\mathrm{deg}]$ & $R e_{D} \times 10^{6}$ & $x_{t} / D$ & $R e_{2, x_{t}}$ \\
\hline 2 & 0 & 1.75 & 0.75 & 0.82 \\
2 & 10 & 1.74 & 0.73 & 0.80 \\
2 & 15 & 1.74 & 0.70 & 0.78 \\
2 & 20 & 1.75 & 0.67 & 0.76 \\
2 & 20 & 2.36 & 0.52 & 0.83 \\
3 & 0 & 2.59 & 0.80 & 0.78 \\
3 & 10 & 1.97 & 0.95 & 0.78 \\
4 & 10 & 2.88 & 1.03 & 0.77 \\
\hline
\end{tabular}

Table 1. Shear layer transition location

\section{Particle image velocimetry results}

Raw PIV recordings are shown in figure 8 , in case of the $0^{\circ}$ model there are no particles present in the wake making it impossible to perform a flow investigation by means of PIV in this region. In the shoulder region the flow undergoes large accelerations as it expands. Due to their inertia the tracer particles slip, see, ${ }^{4}$ which has two effects, it causes the particles to lag with respect to the surrounding flow and the particle streamlines are shifted with respect to the flow streamlines. The particle streamline is always shifted towards regions with lower accelerations. In case of the expanding flow over the shoulder it means that they are shifted outward, preventing particles to enter the wake.

The extent of particle slip is quantified by the particle time relaxation $\tau_{p}$, which is the time constant in 

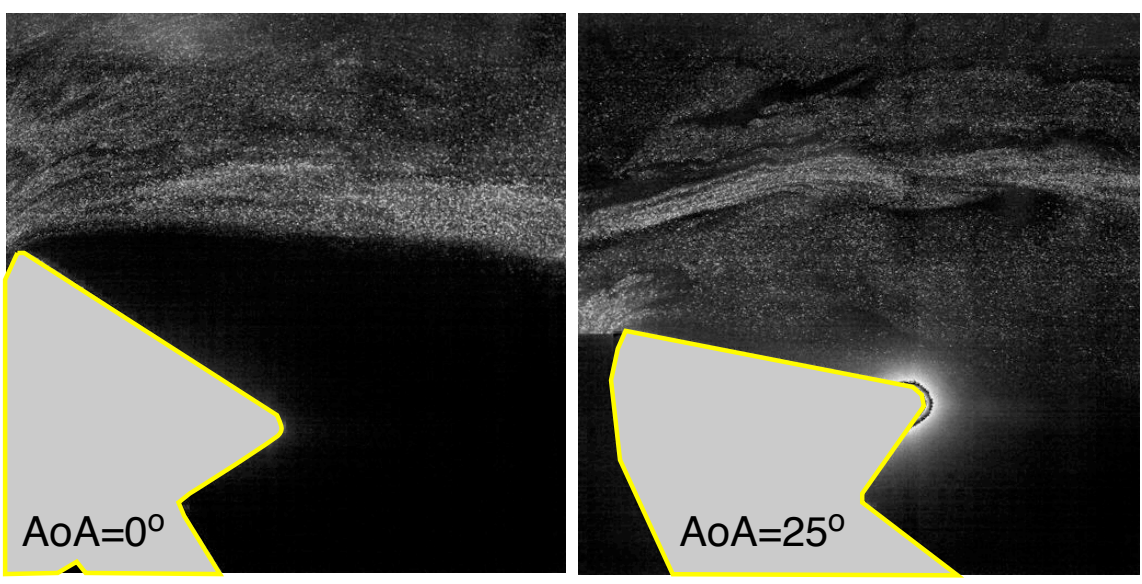

Figure 8. PIV recordings for the $0^{\circ}$ and $25 \circ$ models at Mach 2

the simplified particle motion expression: ${ }^{5}$

$$
\frac{\mathrm{d} U_{p}}{\mathrm{~d} t}=\frac{U_{f}-U_{p}}{\tau_{p}}
$$

where $U_{p}$ is the particle velocity and $U_{f}$ is the flow velocity.

PIV measurements were performed in the Hypersonic Test Facility Delft ${ }^{6}$ at Mach 7 for which the particle slip effect was even more pronounced. For the tracer particles used in the experiment, solid $\mathrm{TiO}_{2}$, the $\tau_{p}$ was determined experimentally by means of a shock wave test ${ }^{7}$ and the value was $\tau_{p}=2.5 \mu s$. In Figure 9 an example PIV recording is given. Overlaid is a laminar CFD solution that is used to compute the particle paths for $\tau_{p}=2.5 \mu s$ (red) and $\tau_{p}=0 \mu s$ (yellow) where the latter is equivalent to a streamline.

From the local particle concentration, the bow shock is clearly visualized as a bright region (the particle density increases with the thermodynamic density). As can be seen from the image, the location of the bow shock indicated by the increase in particle density does not match the shock location obtained from CFD calculations. This is caused by the particle relaxation, since it takes time before the particles decelerate to the post-shock velocity also the particle concentration will increase further downstream.

Near the capsule shoulder the particle concentration decreases again, this is caused by the decrease in thermodynamic density and the effect of particle slip. For the current particles a trajectory is computed starting from a surface streamline. According to the computation, particles are expected above the red line while no particles are expected below. As can be seen from the image, the prediction agrees very well with the measurement, almost no particles are detected below the red particle path.

For the experiments at Mach 2, DEHS was used. For these particles also shock wave tests were performed and a relaxation time of $\tau_{p}=2.1 \mu \mathrm{s}$ resulted. ${ }^{8}$ As may be seen from Figure 8, also this value is still too large.

\section{IV.A. Capsule at $0^{\circ}$ angle of attack}

In figure 10 on the bottom-right a schlieren visualization of the flow around the $0^{\circ}$ capsule is shown. The bow shock is clearly visualized as well as the expansion over the model shoulder. As can be observed from the image, the flow over-expands and a lip shock is formed. Downstream of the shoulder the separated shear layer develops and it does not reattach on the model. In the back of the image the bow shock reflection on the wind tunnel window is visible.

The stereo PIV (SPIV) results are given in the symmetry plane $(z=0 \mathrm{~mm})$ and two horizontal cuts. The expansion from the low velocity region downstream of the bow shock over the model shoulder is clearly visualized. When the $u, v$ and $w$ velocity components at the front of the model are regarded with respect to $z$, the three dimensionality of the flow is apparent; in the front the $w$ component increases with $z$ and the $u$ component decreases. In the 'far' wake region the reflected bow shock coming from the wind tunnel wall is measured. Also the shock coming from the re-compression in the capsule wake is clearly visualized. 


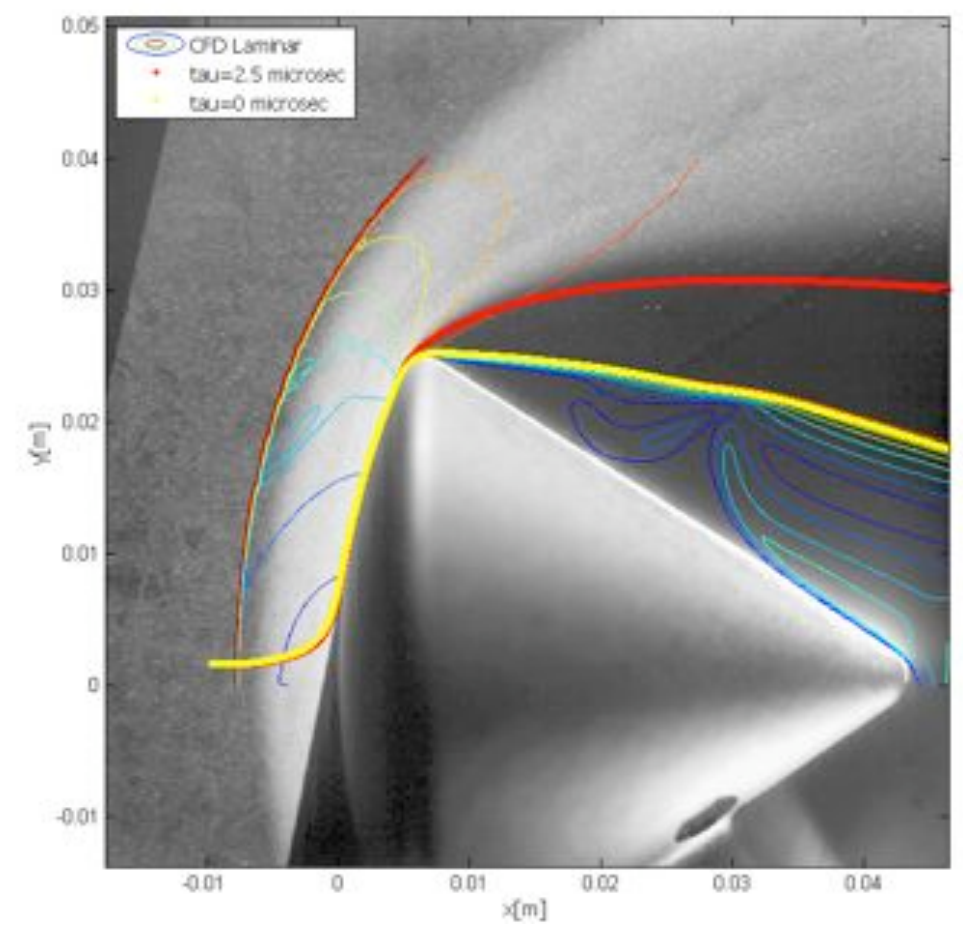

Figure 9. PIV recording at Mach 7 including particle paths
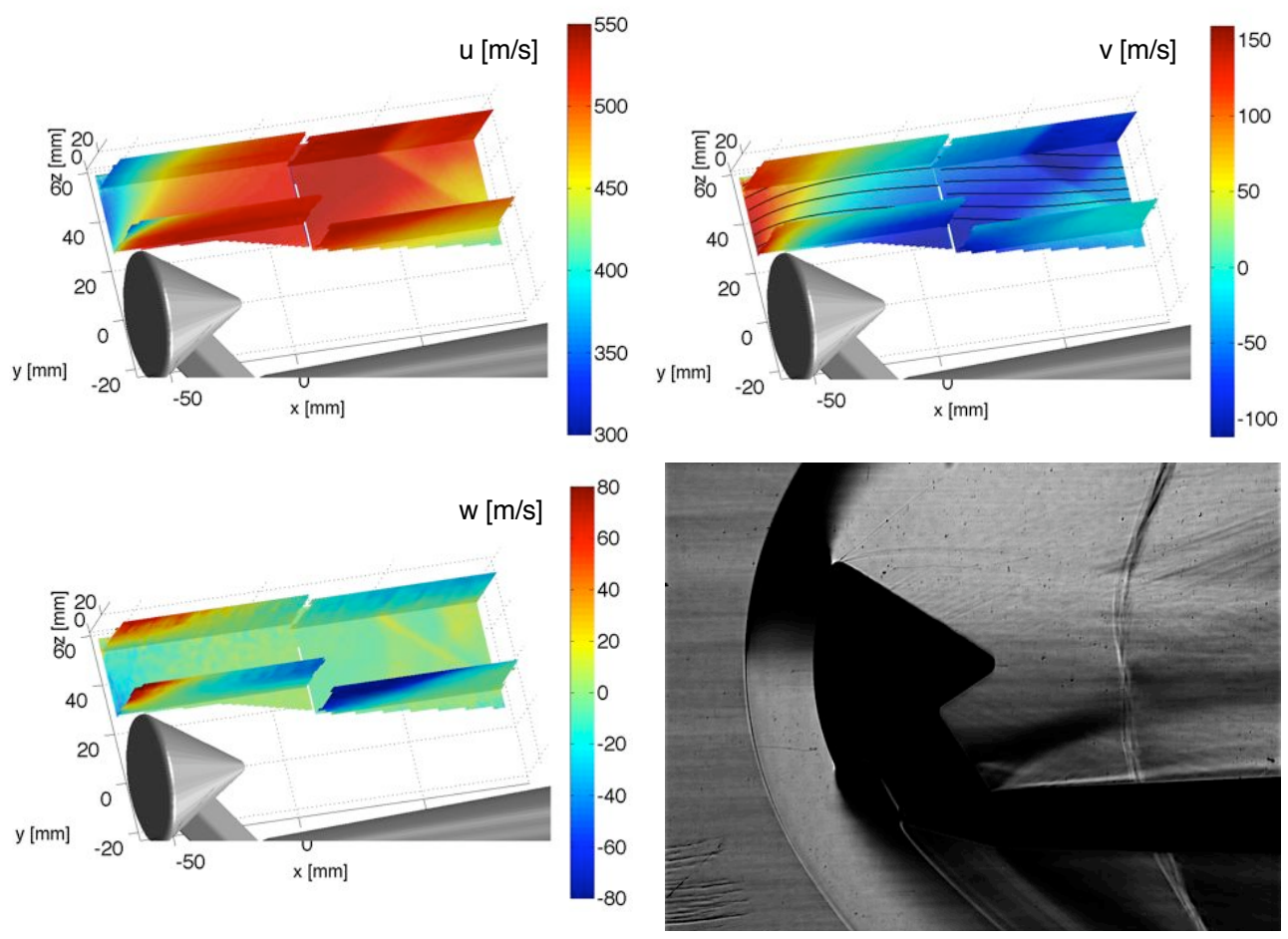

Figure 10. SPIV results and schlieren image of the $0^{\circ}$ model at Mach 2 and $\operatorname{Re}_{D}=1.75 \times 10^{6}$

\section{IV.B. Capsule at $25^{\circ}$ angle of attack}

The PIV and schlieren results for the $25^{\circ}$ model are given in figure 11 . The overall flow structure looks similar to the $0^{\circ}$ case however it can be observed that the shear only partially separates from the upper 
side of the capsule. At the model shoulder a small shock wave is present where separation occurs. Further downstream, approximately halfway the model, a stronger shock is formed where the shear layer reattaches. Downstream of the capsule a strong shock is present where the wake is re-compressed. The shock emanating from the reattaching shear layer is also captured by the PIV measurements (the $u$ component decreases and $v$ component increases). Furthermore the velocity field gives a good overview of the three dimensional flow field directly behind the capsule. The 'far' field clearly shows the three dimensional pattern of reattachment shock that emanates from the wake behind the capsule.
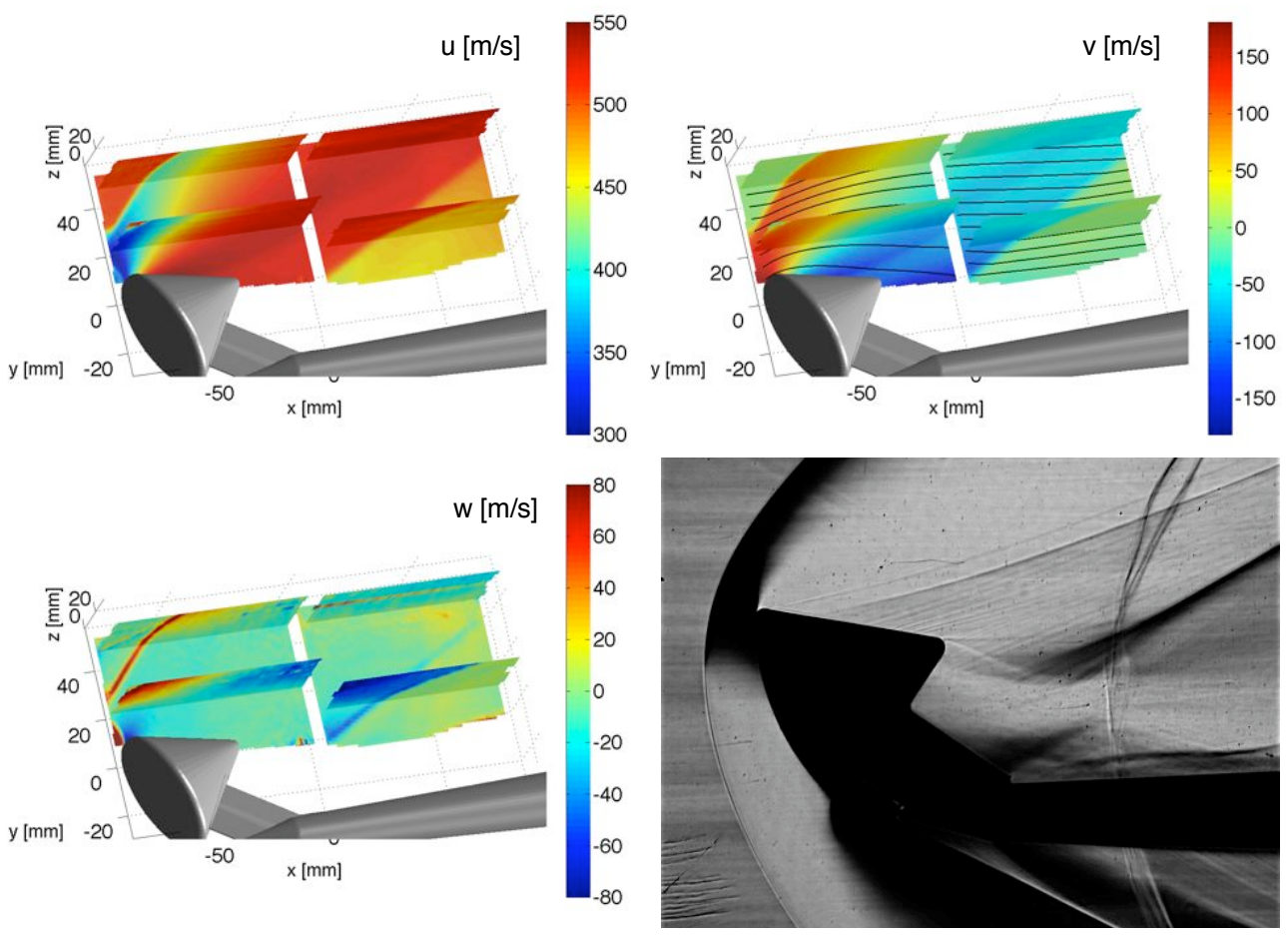

Figure 11. SPIV results and schlieren image of the $25^{\circ}$ model at Mach 2 and $\operatorname{Re}_{D}=1.75 \times 10^{6}$

\section{Conclusion}

The flow over an Apollo type capsule is investigated with the emphasis on the separated shear layer and wake region in the framework of the working group RTO WG043. The model geometry is a scaled version of the AS-202. The state of the shear layer (separated or attached) was observed using shadowgraphy and it was found that the angle of attack for which the shear layer separates decreases with increasing Mach number, furthermore it was observed that the Reynolds number has no appreciable effect on this.

The location where the shear layer undergoes transition from laminar to turbulent was obtained from the shadowgraphs. It was found that the Reynolds number based on the transition location (from stagnation point to transition point) and post normal shock flow conditions was constant: $R e_{2, x_{t}}=0.8 \times 10^{6}$.

Stereo PIV measurements are performed to measure the three dimensional flow field around the capsule at Mach 2 for $0^{\circ}$ and $25^{\circ}$ angles of attack. Two $8 \times 8 \mathrm{~cm}^{2}$ fields of view are considered ('near' and 'far' wake) and the planes are offset at $11 z$ locations. The full three dimensional average velocity field is constructed by combining the measurement planes. Furthermore schlieren visualizations are performed as a complementary measurement technique.

The results for the $0^{\circ}$ capsule give a good overview of the three dimensional flow structure. The shear layer emanating for the capsule shoulder was found to be fully separated. Recompression of the wake occurs downstream of the capsule and the recompression shock is captured in the velocity field. For the $25^{\circ}$ capsule, the shear layer was found to be only partially separated and shear layer reattachment occurs approximately halfway the capsule. This was found in both the schlieren visualization and the PIV results, however in case of the PIV results the three dimensional structure of the separation shock is captured. 


\section{References}

${ }^{1}$ Wright, M., Prabhu, D., and Martinez, E., "Analysis of Apollo Command Module Afterbody Heating Part I: AS-202," Journal of Thermophysics and Heat Transfer, Vol. 20, No. 1, 2006.

${ }^{2}$ Prasad, A., "Stereoscopic particle image velocimetry," Experiments in Fluids, Vol. 29, 2000, pp. 103-116.

${ }^{3}$ Kruse, R., "Transition and flow reattachment behind an Apollo-like body at Mach numbers to 9," Technical note D-4645, NASA, 1968.

${ }^{4}$ Schrijer, F. and Scarano, F., "Particle slip compensation in steady compressible flows," 7 th International Symposium on Particle Image Velocimetry, Rome, Italy, 2007.

${ }^{5}$ Melling, A., "Tracer particles and seeding for particle image velocimetry," Meas. Sci. Technol., Vol. 8, 1997, pp. 1406 1416.

${ }^{6}$ Schrijer, F. and Bannink, W., "Description and Flow Assessment of the Delft Hypersonic Ludwieg Tube," 26th AIAA Aerodynamic Measurement Technology and Ground Testing Conference, No. AIAA-2008-3943, 2008.

${ }^{7}$ Schrijer, F., Scarano, F., and van Oudheusden, B., "Application of PIV in a Mach 7 double-ramp flow," Experiments in Fluids, Vol. 41, 2006, pp. $353-363$.

${ }^{8}$ Ragni, D., Schrijer, F., van Oudheusden, B., and Scarano, F., "Assessment of particle tracers time response by the shock wave test and PIV," Sixth International symposium on PIV, 2009. 